\title{
Human chorioretinal biopsy under controlled systemic hypotensive anaesthesia
}

\author{
I. J. CONSTABLE, G. H. CHESTER, R. HORNE, AND J. F. HARRIOTT \\ From the University of Western Australia and Royal Perth Hospital, Perth, \\ Western Australia
}

SUMMARY This paper describes a simplified technique for biopsy of the retina and choroid which had been used in 5 human volunteers. The biopsy was carried out in 4 immediately before enucleation of an eye for malignant melanoma and in 1 patient who was undergoing trabeculectomy for painful glaucoma associated with retinitis pigmentosa. A combination of intravenous mannitol and transient controlled systemic hypotension, induced under general anaesthesia with intravenous sodium nitroprusside, was used in 3 cases and resulted in no vitreous loss and minimal bleeding. In the 2 cases in which hypotension was not used bleeding was a definite problem, but no vitreous loss was experienced.

Understanding of many types of chorioretinal pathology has long been retarded by the lack of a practical biopsy technique. Previous workers have described a full-thickness eye wall method for biopsy of the posterior segment in rabbits, monkeys, and 1 human. ${ }^{1-3}$ A transvitreal approach to retinal biopsy has also been reported in animals. ${ }^{4}$ These methods have not been widely adopted because of the complexity of the procedure and of the universal vitreous loss reported. A simplified trans-scleral approach to chorioretinal biopsy in dogs using hypotensive anaesthesia resulted in minimal or no vitreous loss, ${ }^{5}$ so that we were encouraged to apply the procedure to 5 human volunteers.

\section{Patients and methods}

CASE 1

A 35-year-old male presented to the neurology outpatients with a 6-week history of blurred vision in the left eye. Vision was 6/6 right, counting fingers left, and a superior field defect was noted in the left eye. On referral to the ophthalmology department a large serous detachment of the inferior retina in the left eye was noted as well as an underlying pigmented mass that had the clinical characteristics of a large melanoma of the choroid. Fluorescein angiography, ${ }^{32} \mathrm{P}$ isotope testing, and ultrasonography all suggested a melanoma of the

Correspondence to Professor I. J. Constable, University Department of Ophthalmology, Royal Perth Hospital, Wellington Street, Perth 6000, Western Australia. choroid. The lesion was judged to be too large for local radiotherapy or photocoagulation and enucleation was advised. A systemic survey for metastases was negative. Appropriate patient consent was obtained. A local chorioretinal biopsy in the upper temporal quadrant in the area of attached retina was planned immediately before enucleation of the patient's left eye. A spindle cell B melanoma was confirmed.

CASE 2

A 45-year-old female was referred with a 3-month history of blurred vision in the right eye in August 1978. Her general health had been previously excellent. On examination there was a large collarstud mass occupying the midperipheral area of the inferior retina. It was pigmented and approximately 6 disc diameters in size. There was a surrounding serous detachment which extended to the fovea. Fluorescein angiography, ${ }^{32} \mathrm{P}$ uptake, and ultrasound were all positive for melanoma. Vision was reduced to $6 / 12$ right eye. The left eye had $6 / 5$ vision and no abnormalities. The mass was considered too large for local treatment, and enucleation of the right eye was recommended. On 8 September 1978, with appropriate patient consent, a chorioretinal biopsy was carried out before right enucleation. Histology confirmed an epithelioid melanoma of the choroid.

\section{CASE 3}

A 45-year-old man presented to the ophthalmology department in February 1979 with a 4-month history of blurred vision centrally in the left eye. 
His general health had been previously excellent. Vision was $6 / 5$ right, $6 / 60$ left. There was a large raised pigmented mass 4 disc diameters in area at the posterior pole of the left eye. It was surrounded with a thin layer of subretinal fluid. Fluorescein angiography and ${ }^{32} \mathrm{P}$ uptake were both positive for melanoma. No evidence of systemic metastases was found on routine surveys. Enucleation was recommended for the left eye because of the size, position, and surrounding subretinal fluid associated with the tumour. Permission was obtained for chorioretinal biopsy, and this was carried out under general anaesthesia immediately before enucleation on 14 March 1979. A spindle cell B melanoma was confirmed.

\section{CASE 4}

A 70-year-old man was found to have a raised pigmented mass in the inferonasal quadrant of the right eye on routine examination by his ophthalmologist in 1974. This was followed at regular intervals until May 1979, when the patient presented with sudden loss of vision in the right eye associated with vitreous haemorrhage. The patient had been admitted to hospital with a myocardial infarct in January 1978 but had made an excellent recovery and has now considered to be in reasonable general health. Enucleation of the right eye was advised after consultation with several ophthalmologists, and permission was obtained to carry out a chorioretinal biopsy immediately before this procedure. In this case controlled hypotensive anaesthesia was withheld because of the patient's age and history of ischaemic heart disease. Pathology confirmed a spindle cell B melanoma.

CASE 5

A 68-year-old woman presented to the ophthalmology outpatient department in July 1978 with a painful blind right eye and vision reduced to counting fingers in the left. The patient had a history of retinitis pigmentosa with poor night vision and constricted visual fields by the age of 20 . There was no family history of the disease. The patient was declared legally blind in 1968 at the age of 57 and was admitted as a chronic patient to the Braille hospital. She had a 5 year history of mild maturity onset diabetes and was also on digoxin and diuretic for congestive cardiac failure. On examination the right eye had marked central corneal oedema, a narrowed anterior chamber, and a closed angle. The lens showed moderate nuclear sclerosis but no signs of intumescence. No view was obtained of her fundus. Intraocular pressure was $50 \mathrm{mmHg}$ right, $15 \mathrm{mmHg}$ left eye. The left eye showed nuclear sclerosis of the lens and end-stage retinitis pigmen- tosa. On gonioscopy the left angle was narrow but open. The patient was treated for 3 months with pilocarpine drops and systemic acetazolamide. She continued to have intermittent pain in the right eye and was often distressed with vomiting. She was admitted to hospital on 29 September 1978 with congestive cardiac failure. This responded to increase in the diuretic regimen, and the patient was again discharged. Treatment for the chronic glaucoma in the right eye was continued, but pain and discomfort did not subside. The patient was then readmitted on 29 November 1978, at which time the blind right eye was still painful. There was persistent right corneal oedema. Intraocular pressure was $50 \mathrm{mmHg}$ right, $12 \mathrm{mmHg}$ left. On 1 December 1978 a right trabeculectomy was planned for the chronic closed-angle glaucoma. Permission was obtained from the patient to carry out a chorioretinal biopsy at the same time. Again no hypotensive anaesthesia was used. On the third postoperative day the patient developed intermittent paroxysmal tachycardia and signs of pulmonary oedema. Despite therapy she failed to respond and died on the fourth postoperative day.

\section{ANAESTHETIC TECHNIQUE}

In cases 1,2 , and 3 intravenous mannitol $1 \mathrm{~g} / \mathrm{kg}$ was given 20 minutes before retinal biopsy to soften the eye and decrease the vitreous volume. At the time of biopsy transient systemic hypotension was also induced. Preoperative anaesthetic assessment excluded cardiovascular and respiratory disease. The electrocardiogram was normal in these 3 cases, as was blood count and chext $x$-ray. Premedication was confined to $25 \mathrm{mg}$ promethazine $\mathrm{HCl}$ intramuscularly 1 hour before induction of anaesthesia. Standard anaesthetic induction included preoxygenation, thiopentone $300 \mathrm{mg}$ intravenously, muscle paralysis with intravenous suxamethonium pretreated with D-tubocurarine, and lignocaine spray to the vocal cords. A cuffed Oxford tube was placed in position, and anaesthesia was maintained with nitrous oxide 3 litres/minute, oxygen 1.5 litres/ minute, and $1-2 \%$ halothane on a semiclosed circuit. The ECG was monitored continuously, and brachial blood pressure was monitored on both arms with an oscillometer. An intravenous line for infusion of mannitol was placed in the left arm; a second intravenous line was placed into a dorsal vein in the left foot for infusion of sodium nitroprusside. This latter intravenous drip was set up with $30 \mathrm{mg}$ sodium nitroprusside in $500 \mathrm{ml}$ of $5 \%$ dextrose. A trial dose of sodium nitroprusside was infused early in the operation to test the patient's individual response. In case 1 it was found that systolic blood pressure was difficult to lower 


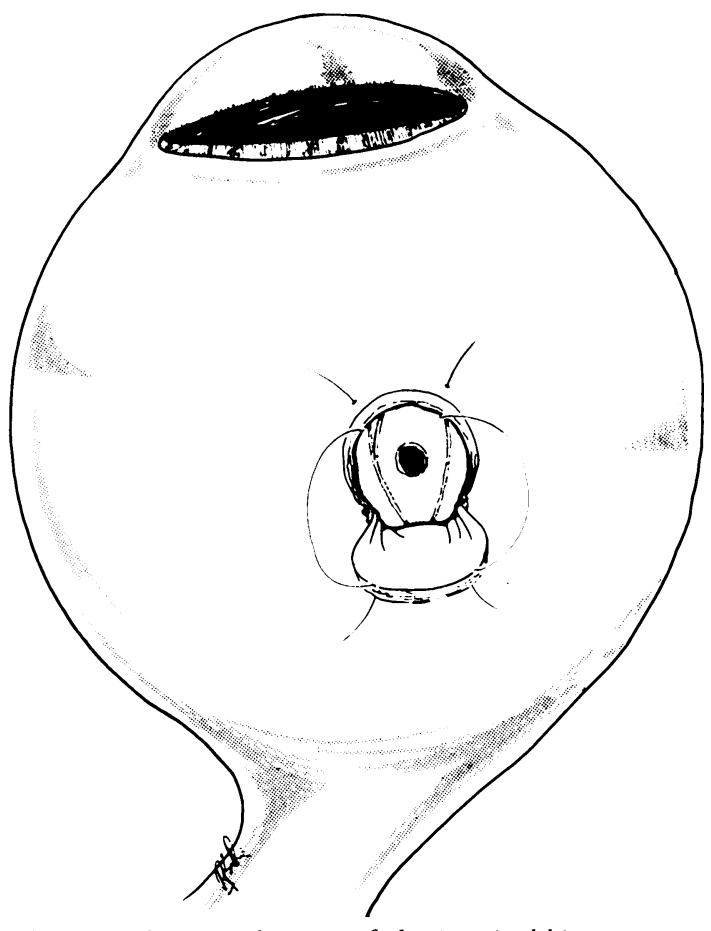

Fig. 1 Schematic drawing of chorioretinal biopsy site in a human eye. below $80 \mathrm{mmHg}$ owing to a persistent tachycardia of $90 /$ minute. The patient was therefore given practolol $1 \mathrm{mg}$ intravenously, which reduced the pulse rate to $65 /$ minute. Immediately before the final steps in the chorioretinal biopsy the sodium nitroprusside infusion was allowed to drip slowly so that the systolic blood pressure was lowered to $70 \mathrm{mmHg}$ over $2 \frac{1}{2}$ minutes. This level was maintained with continuous infusion over 5 to 10 minutes during which time the biopsy was taken. A total of 60 to $80 \mathrm{ml}$ of infusion containing 3.6 to $4.8 \mathrm{mg}$ sodium nitroprusside infused at the rate of approximately $8 \mu \mathrm{gm} / \mathrm{kg} /$ minute was used in each case. At the end of the biopsy the sodium nitroprusside drip was turned off, and blood pressure returned to normal levels under anaesthesia within 3 minutes.

In cases 4 and 5 , because of age and history of cardiovascular disease, intravenous mannitol and sodium nitroprusside infusion were withheld. In both cases the general anaesthesia was induced with $150 \mathrm{~g}$ of thiopentone after preoxygenation, and intubation was carried out after $10 \mathrm{mg}$ suxamethonium and lignocaine spray to the vocal cords. Maintenance was with a nitrous oxide/oxygen mixture in a $3: 1$ ratio. Intermittent enflurane, droperidol (total $5 \mathrm{mg}$ ), phenoperidine $(0.5 \mathrm{mg})$, and alcuronium chloride (10 $\mathrm{mg}$ ) were given during the procedure. Acetazolamide $(500 \mathrm{mg})$ was given

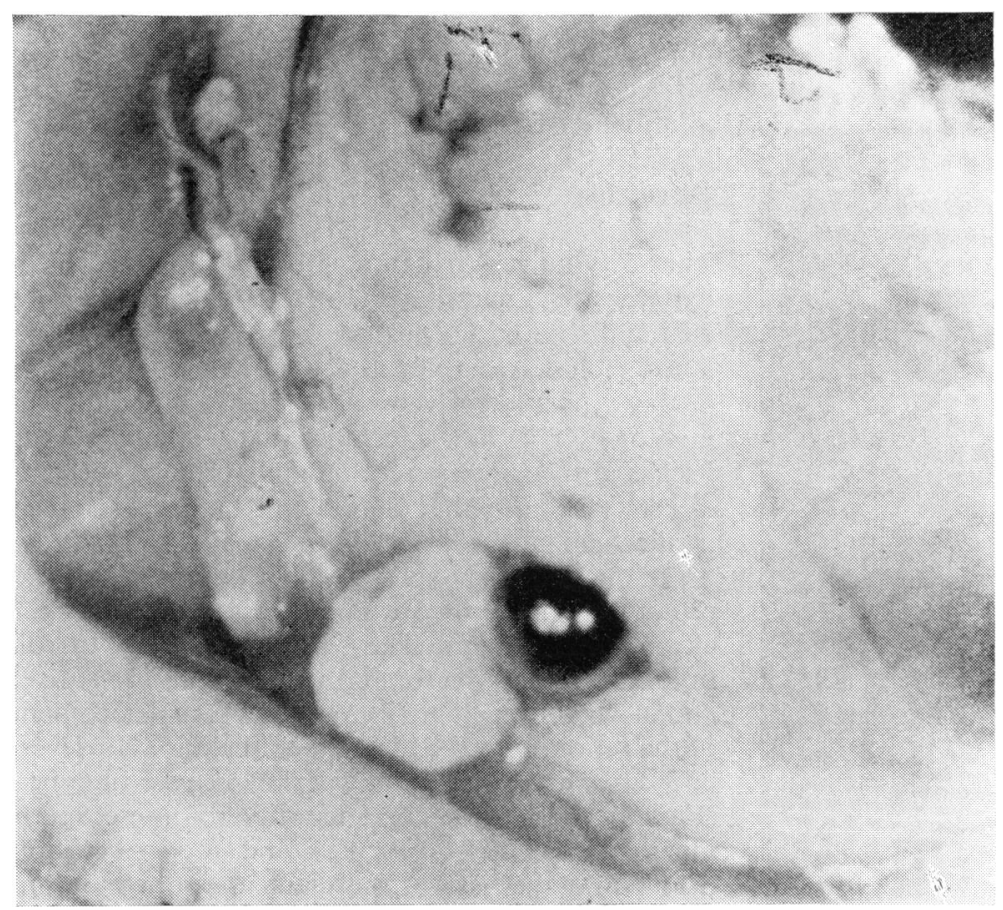

Fig. 2 Intact vitreous face after chorioretinal biopsy. Print taken from a $16 \mathrm{~mm}$ film. 
intravenously to lower intraocular pressure during the procedure.

\section{SURGICAL TECHNIQUE}

After conjunctival peritomy and placement of bridle sutures a biopsy site was selected in the superior temporal quadrant posterior to the equator, except in case 3, in which an upper nasal site was selected. In each case the site selected was overlying attached retina on the opposite side of the globe to the melanoma.

The exact site was chosen clear of major vortex

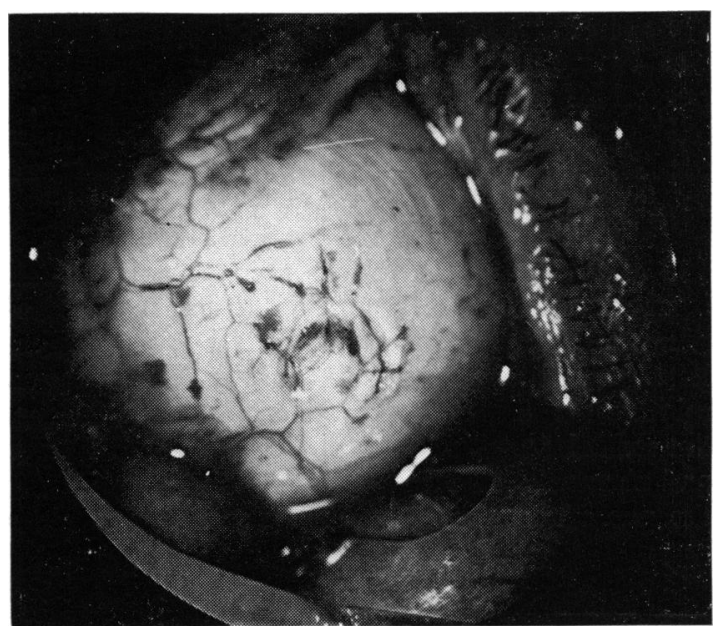

Fig. 3 Full-thickness scleral flap closed with interrupted 8-0 Vicryl sutures after chorioretinal biopsy.

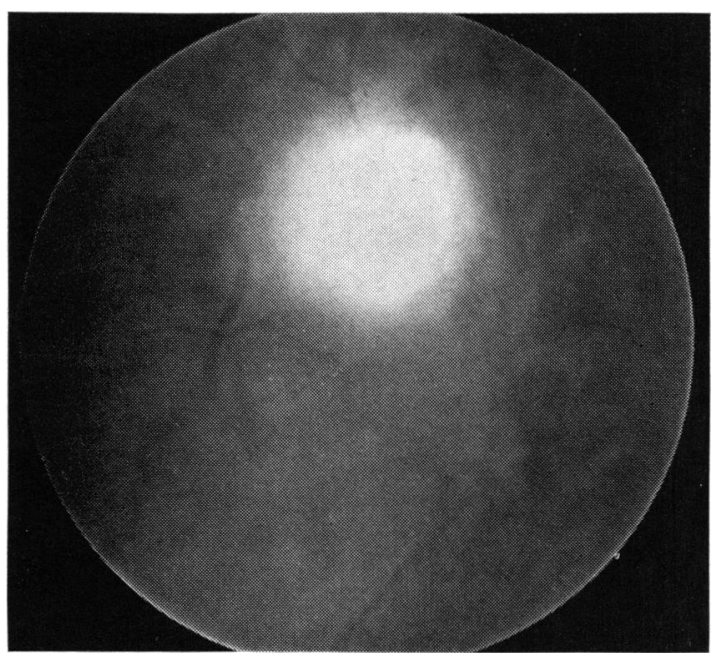

Fig. 4 Fundus photography of biopsy site immediately after closure of the scleral flap and prior to enucleation of the eye in case 1. There is no evidence of bleeding and no elevation of the surrounding retina. veins after transillumination of the globe. A fullthickness, circular, $270^{\circ}$ scleral flap of $3 \mathrm{~mm}$ diameter was then raised (Fig. 1). Two 8-0 Vicryl sutures were placed through the flap and the host bed of sclera to facilitate closure after chorioretinal biopsy. The site was again transilluminated to select the area for biopsy in between larger choroidal vessels. At this stage the sodium nitroprusside infusion was started in the first 3 cases described and this visibly blanched the choroid. The eye was already soft owing to infusion of mannitol $1 \mathrm{~g} / \mathrm{kg}$ intravenously over the previous 20 minutes. When systolic blood pressure was reported by the anaesthetist to be lowered to a level of $70 \mathrm{mmHg}$, a biopsy was taken with blunt jeweller's forceps and small curved Vannas scissors. The choroid and underlying retina were slightly tented with the grasp of the forceps, and the first cut exposed the vitreous face. After inserting one blade along the vitreous face the biopsy was excised with 2 more cuts. It was immediately placed in glutaraldehyde and the instruments were changed for closure of the wound. In all 5 cases the vitreous face bulged, but none was lost (Fig. 2). In cases 4 and 5, in which mannitol and hypotensive anaesthesia were withheld, there was excessive choroidal bleeding, which meant that a fragmented biopsy was obtained. The scleral flap was closed with the preplaced sutures (Fig. 3). In cases 1 to 4 before enucleation of the globe photographs were taken of the biopsy site with a portable Kowa fundus camera (Fig. 4). In case 5 the eye was not removed but a routine trabeculectomy was carried out in the superior temporal quadrant under a limbal based scleral flap.

\section{Discussion}

The 3 younger patients submitted to transient hypotensive anaesthesia tolerated the procedure well and showed no postoperative side effects. Case 5 unfortunately developed acute pulmonary oedema 4 days postoperatively and died. After careful investigation it was concluded that the anaesthetic itself and the surgery were unlikely to have been the direct cause, but that they might have contributed to the final cardiovascular decompensation.

Of the 5 biopsy specimens of the choroid and retina obtained only 3 were entirely satisfactory for electron microscopy. The specimens tended to roll up on the end of the forceps when placed in fixative, and it was difficult to prevent artefact detachment of the retina from the pigment epithelium. However, in 3 cases good individual specimens were obtained (Fig. 5). High power examination of these specimens confirmed good fixation and good resolution of detail at the base of the pigment epithelium and 


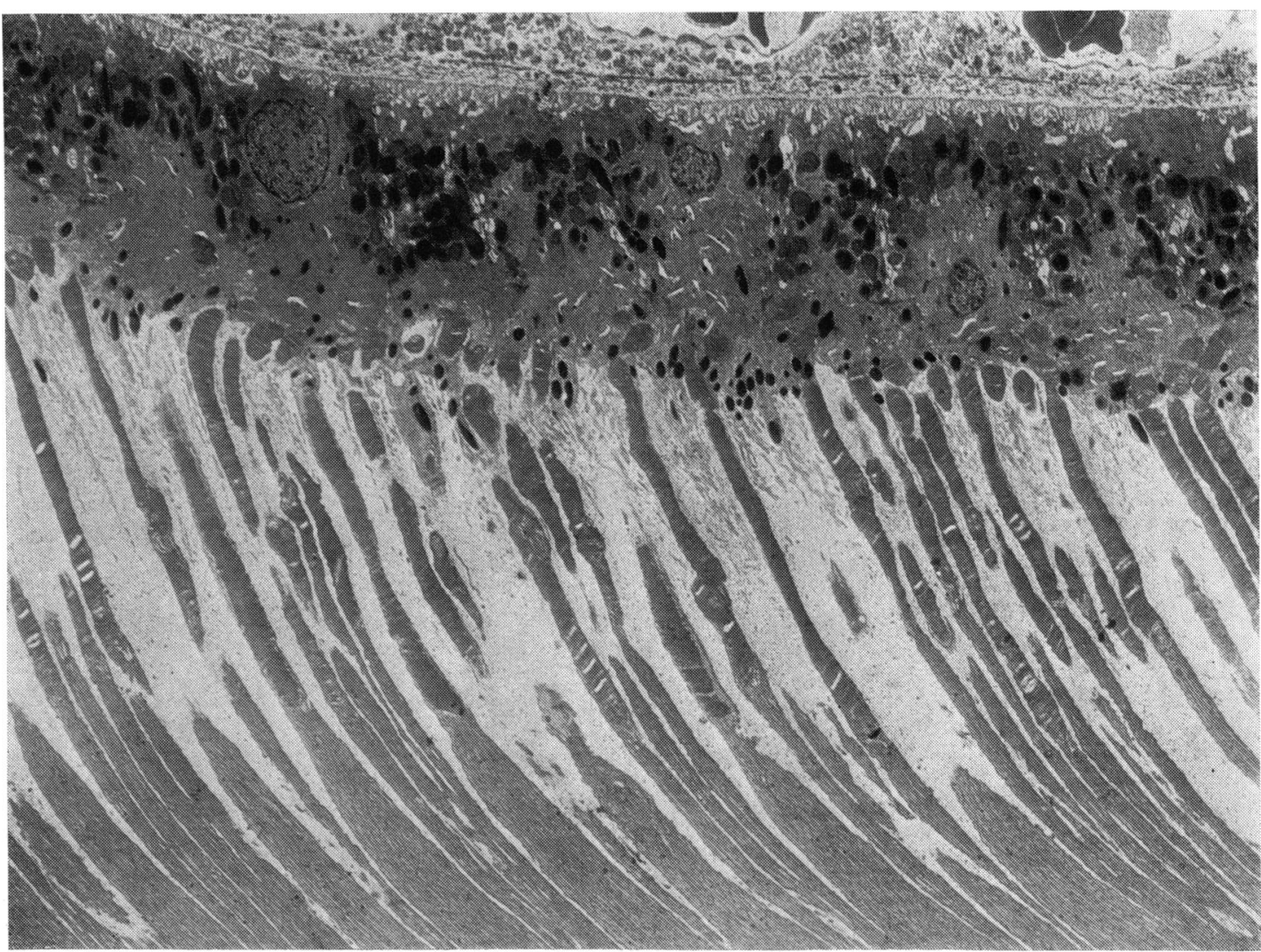

Fig. 5 Electron microscopic appearance of chorioretinal biopsy from case 1 showing some bending of outer segments and slight spreading of the receptor discs. The specimen is quite adequate for interpretation. $(\times 1880$.

Bruch's membrane, the receptors and the internal limiting membrane of the retina respectively (Fig. 6). The poor specimens were obtained from the 2 eyes in which hypotensive anaesthesia was not employed. Because of the bleeding it was not possible neatly to excise the biopsy specimens, and indeed they were removed piecemeal because of the risk of cutting into the vitreous face in the presence of blood. However, individual cell layers could be assessed in these 2 specimens. It is to be noted that renal and liver biopsies are often less then ideal.

The choroidal bleeding was surprising in the case of the eye with end-stage retinitis pigmentosa. It had been presumed that, because of choroidal and retinal atrophy, bleeding would not be a problem.

Nevertheless even in the cases not subjected to ocular and systemic hypotension it was shown that choroid and retina could be excised without losing vitreous. It is suggested that, if the problems of handling such minute specimens of choroid and retina can be overcome, this technique may have widespread application, particularly in young patients in whom controlled hypotensive anaesthesia can be employed without significant risk. In this context it is to be noted that sodium nitroprusside infusion is used to induce transient hypotension in a wide variety of surgical fields, notably vascular, cerebral, and cardiac surgery. In these areas the hypotension is often maintained for long periods without ill effects. In the case of retinal biopsy it is necessary for only 5 to 10 minutes.

It is obviously of great importance to determine whether excision of a $1 \mathrm{~mm}$ piece of choroid and retina without disturbance of the vitreous face would lead to retinal detachment in humans in the short term or long term. To date, the definitive experiment, which would require a volunteer to submit a blind eye perhaps from optic atrophy to the procedure, has not been carried out, It is noteworthy, however, that retinal detachment must be extremely rare in patients with retinal dystrophies, as we have been unable to extract any reports from the literature or from anecdotal sources to date. It is possible in those patients who have widespread pigment 
Fig. 6 Composite higherpower electron micrographs of human chorioretinal biopsy specimen. (A) Basal section of retinal pigment epithelial (rpe) cell $(\times 8640)$. (B) Apical section of rpe cell $(\times 7808)$. (C) Junction of inner and outer segments of rods $(\times 10000)$. (D) Inner surface of retina showing internal limiting membrane ( $\times 11200)$.
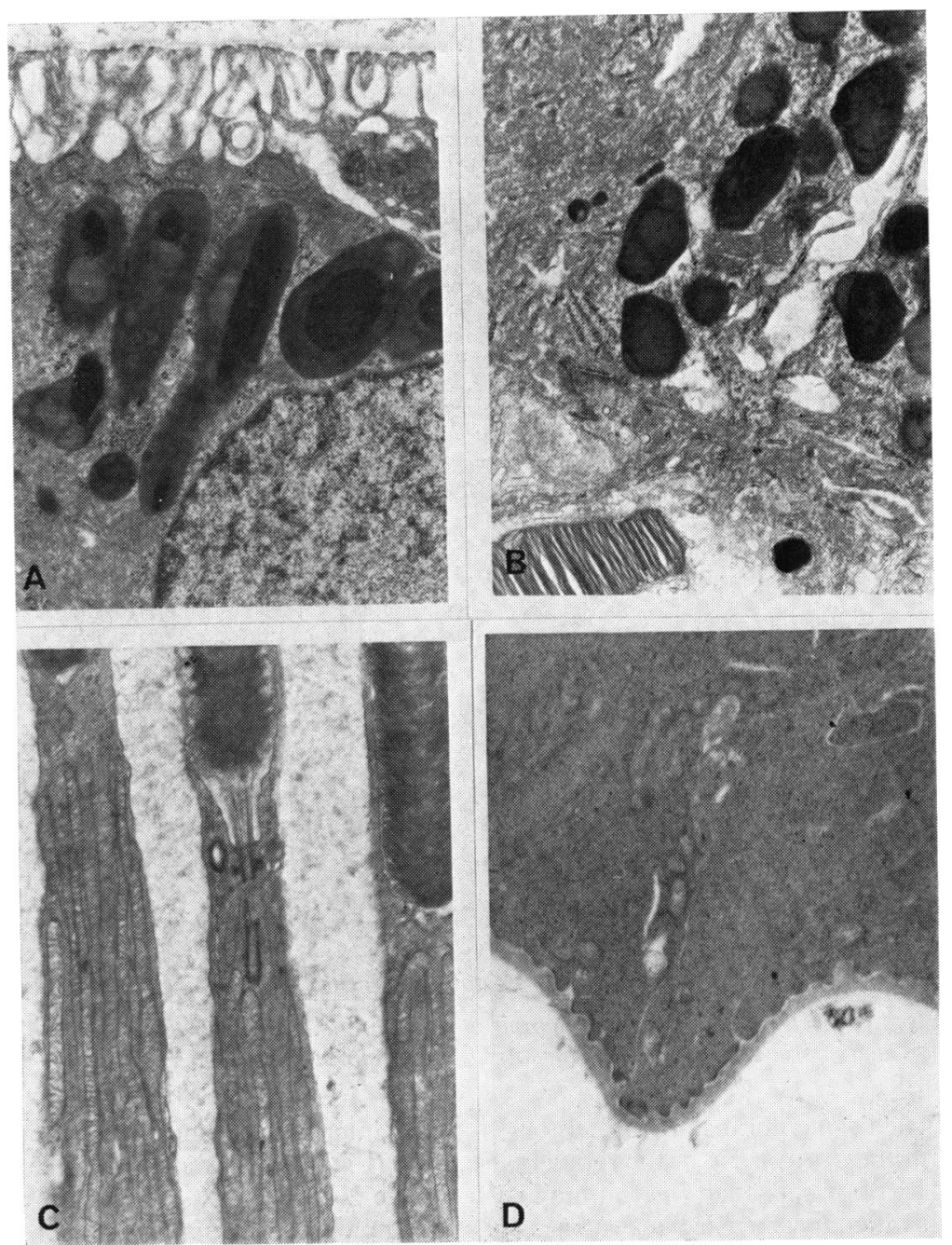

epithelial disturbances that the risk of retinal detachment is minimal because of pathological retinal adhesion.

If this simplified chorioretinal biopsy technique can be shown in a large number of cases to be free of the dual risks of massive haemorrhage and significant vitreous loss, then a wide range of laboratory techniques could be employed to elucidate chorioretinal diseases at present poorly understood.

We thank Dr D. C. Wilson for referring two of the cases, Mr C. Barry for clinical photography and Miss H. Deady for secretarial help.

\section{References}

${ }^{1}$ Peyman GA, Meisels HI, Batko KA, Vlchek JK. Fullthickness eye wall biopsy. I. An experimental approach to the tissue diagnosis and study of choroidal and retinal lesions. Invest Ophthalmol 1975; 14: 484-7.

${ }^{2}$ Peyman GA, Homer P, Kasbeer R, Vlchek J. Full thickness eye wall biopsy. II. In primates. Invest Ophthalmol 1975; 14: $565-7$.

${ }^{3}$ Peyman GA, Fishman GA, Sanders DR, Apple DJ, Vlchek JK. Biopsy of human scleral-chorioretinal ti-sue. Invest Ophthalmol 1975; 14: 707-10.

${ }^{4}$ Griffin JR, Straatsma BR, Kreiger AE. Transvitreal chorioretinal biopsy in the rabbit. Am J Ophthalmol 1975; 79: 25-38.

${ }^{5}$ Constable IJ, Slatter DH, Horne R. Chorioretinal biopsy in dogs. Invest Ophthalmol 1979; 19: 603. 